\title{
Féeries
}

Études sur le conte merveilleux, XVII $-\mathrm{XIXe}$ siècle

$17 \mid 2021$

\section{Contes en couleur}

\section{Des personnages hauts en couleur?}

Les usages des couleurs dans les Romans et contes de voltaire

Colorful Characters? The Uses of Color in Voltaire's Novels and Tales

\section{Olivier Ferret}

\section{(2) OpenEdition}

Journals

Édition électronique

URL : https://journals.openedition.org/feeries/3749

DOI : $10.4000 /$ feeries.3749

ISSN : $1957-7753$

Éditeur

UGA Éditions/Université Grenoble Alpes

Édition imprimée

ISBN : 978-2-37747-327-4

ISSN : 1766-2842

Référence électronique

Olivier Ferret, « Des personnages hauts en couleur? », Féeries [En ligne], 17 | 2021, mis en ligne le 17 décembre 2021, consulté le 14 janvier 2023. URL : http://journals.openedition.org/feeries/3749 ; DOI : https://doi.org/10.4000/feeries.3749

Ce document a été généré automatiquement le 14 janvier 2023.

Tous droits réservés 


\title{
Des personnages hauts en couleur?
}

\author{
Les usages des couleurs dans les Romans et contes de voltaire \\ Colorful Characters? The Uses of Color in Voltaire's Novels and Tales
}

\section{Olivier Ferret}

1 Depuis les travaux fondateurs de Michel Pastoureau, Manlio Brusatin et John Gage, la réflexion sur le sens et la valeur des couleurs constitue un champ d'étude en plein essor, ce qu'illustrent, entre autres, les contributions réunies par Aurélia Gaillard et Catherine Lanoë dans le dossier thématique de la revue Dix-huitième siècle en 201919. Par son caractère polygraphique, l'œuvre de Voltaire apparait comme un terrain d'observation a priori intéressant. On trouve trace du "renversement newtonien ", étudié par Bertrand Maitte ${ }^{2}$, dans les Éléments de la philosophie de Newton ${ }^{3}$, composés en 1736-1737, dont la première édition est publiée l'année suivante. Indice parmi d'autres de la fluidité avec laquelle les questions circulent dans l'œuvre, l'«histoire philosophique » intitulée Micromégas (1752), élaborée au cours de la même période, fait référence, avec la liberté qu'autorise la fiction, aux « couleurs primitives » qui servent de mesure pour appréhender la diversité des échelles et la leçon de relativisme qu'elle invite à tirer. « De quelle couleur est votre soleil, bien examiné ? ", demande l'habitant de Sirius à celui de Saturne :

- D'un blanc fort jaunâtre, dit le Saturnien; et quand nous divisons un de ses rayons, nous trouvons qu'il contient sept couleurs. - Notre soleil tire sur le rouge, dit le Sirien, et nous avons trente-neuf couleurs primitives ${ }^{4}$.

2 On trouve encore trace d'une approche sensualiste forgée au contact de la philosophie anglaise, par exemple dans le chapitre 4 du Traité de métaphysique, rédigé en 1734 : la couleur est considérée, en référence à John Locke, comme l'un des « modes » d'un objet qui ne sont, déclare le philosophe, "autre chose que mes perceptions", ce qui n'offre pourtant - il faut le souligner - aucune " preuve » que «cette couleur » existe ${ }^{5}$. Dans l'article « IMAGINATION", rédigé pour l'Encyclopédie, sans revenir sur les principes d'une théorie de la connaissance fondée sur le primat de la perception, Voltaire met l'accent sur la place centrale des images: "Ainsi tous vos raisonnements, toutes vos connaissances, sont fondées sur des images tracées dans votre cerveau : [...] ces images sont la base de toutes vos notions ${ }^{6}$. $"$ Il n'est cependant pas question de couleur, 
contrairement à ce que l'on peut lire dans l'article du même titre que signe Marmontel dans le Supplément de l'Encyclopédie: "Quand l'imagination ne fait que retracer les objets qui ont frappé les sens, elle ne diffère de la mémoire que par la vivacité des couleurs ${ }^{7}$. »

Si l'étendue des références est relativement limitée dans les écrits théoriques, le corpus des contes $^{8}$ de Voltaire semble plus prometteur ${ }^{9}$. Prenant acte de l'absence de corrélation constatée, dans l'article de l'Encyclopédie, entre images et couleurs, on concentrera l'attention sur l'évocation des couleurs proprement dites au détriment de la force suggestive des images - souvent sanglantes - qui confèrent aux situations mises en œuvre dans les contes une coloration rouge dominante. Quantitativement peu nombreuses ${ }^{10}$, le simple relevé des occurrences indique qu'elles couvrent, outre le blanc (l'argent), le gris, le noir, et certaines nuances de tons (blond, brun), l'intégralité du spectre chromatique visible: rouge, écarlate, incarnat, vermeil, rose, corail, jaune, (d)or(é), vert, bleu, azur, violet.

4 Afin de réfléchir, dans les limites imposées par cette contribution, à l'économie visuelle qui sous-tend l'exploitation de cette large palette, il a été nécessaire de procéder à des choix. On ne signalera ici que pour mémoire ce qui relève, dans le recours aux codes génériques du conte, en particulier oriental, de la couleur locale, qu'elle confère un degré de vraisemblance minimal au récit ou participe des effets, sinon de parodie, du moins de mise à distance des topiques narratives et descriptives que Voltaire convoque : dans un cadre merveilleux, on ne compte pas les mentions d'un casque, d'un étui, d'une aiguille d'or, ou encore de plats, brochettes et vases d'or qui constituent les accessoires du riche décor de La Princesse de Babylone (1768) ${ }^{11}$; dans une autre veine, qui exploite les lieux communs sur les mœurs des nations, lorsque, dans le même texte, les personnages arrivent en Espagne, il est question d'une «nation, vêtue de noir », qui «semblait en deuil», où «le peuple» comme les "graves personnages" portent «manteau noir » et « fraise $»^{12} \ldots$

Quoique, comme on le voit, le cadre ne soit pas sans rapport avec les personnages, l'enquête va se cantonner à cette échelle : en s'interrogeant sur ces personnages hauts en couleur qui peuplent l'univers des contes de Voltaire, il s'agira de réfléchir aux usages rhétoriques et symboliques des couleurs. Après avoir mis en évidence leur fonction dans l'économie narrative des récits, on s'interrogera sur la dimension idéologique des dispositifs qui les mettent en jeu. On verra ainsi que les modalités de l'exploitation, voire du détournement, dans ces récits, d'une symbolique des couleurs largement héritée conduisent à mettre à distance sinon à déjouer le codage genré de la caractérisation des personnages. On montrera enfin que le langage des couleurs, par delà la dichotomie du noir et du blanc, est aussi mis au service d'un discours philosophique, y compris dans son acception militante.

\section{Les babouches d'Astarté}

6 En tant qu'elles permettent l'identification de personnages au premier coup d'œil, les couleurs sont investies d'une fonction narrative au sein du récit. C'est en particulier le cas pour les personnages (proto)typiques, dont le rôle est souvent accessoire dans le récit et qu'il s'agit de camper de manière efficace. On songe à la fonction des uniformes de la soldatesque identifiables par leur couleur. Ainsi des circonstances de l'enrôlement de Candide parmi les Bulgares : "Deux hommes habillés de bleu le remarquèrent.» Puis, selon une logique métonymique: "Ah! monsieur, lui dit un des bleus, les 
personnes de votre figure et de votre mérite ne payent jamais rien [...]. ${ }^{13}$ Tel n'est pas le cas du «jeune guerrier " que rencontre Babouc dans Le Monde comme il va (1748), à qui il « en a coûté quarante mille dariques d'or [...] pour coucher sur la terre trente nuits de suite en habit rouge ${ }^{14} »$. Cependant, la signification de la couleur de certains habits demeure parfois ambiguë, à l'instar de l'habit noir qui, on l'a vu, peut passer, en contexte ibérique, pour le costume national espagnol. Au début du même chapitre de ce conte, « un homme grave, en manteau noir » se présente, demande à parler à un jeune magistrat qui lui remet des papiers et le congédie sans ménagement. Le mystère de son identité ne fut levé que lorsque "Babouc demanda quel était cet homme » et que «la maitresse de maison lui dit tout bas : "C'est un des meilleurs avocats de la ville [...]" »" Dans ses pérégrinations, Scarmentado croise pourtant en Hollande « un prédicant à manteau noir ${ }^{16}$ ", que l'on peut rapprocher de ce "petit homme noir » que rencontre l'Ingénu lorsque, au cours de son voyage à Versailles, il soupe avec des huguenots : «l'homme noir » lui livre alors le détail des persécutions dont ses coreligionnaires sont victimes depuis la révocation de l'édit de Nantes $^{17}$. Mais l'habit fait aussi le moine, comme l'indiquent, en contexte espagnol, le défilé de l'« armée des moines [...] deux à deux, blancs, noirs, gris, chaussés, déchaussés, avec barbe, sans barbe, avec capuchon pointu, et sans capuchon» auquel assiste Scarmentado en prélude à la scène d'autodafé ${ }^{18}$, et, en contexte oriental, l'évocation $d u$ "grand procès entre les mages blancs et les mages noirs » que Zadig termine « heureusement $»^{19}$, pour ne rien dire des «mages de toutes couleurs ${ }^{20} »$ que Babouc rencontre dans l'antichambre d'un ministre de Persépolis.

7 Si les codes de la reconnaissance sont parfois brouillés, tel ne doit pas être le cas des couleurs faisant office d'emblèmes mettant en œuvre une rhétorique exploitée mais aussi problématisée, dans Zadig, au cours de l'épisode des "combats". Lors de cette première épreuve qualifiante, censée permettre d'identifier celui qui, par sa vaillance, est digne d'accéder au trône de Babylone, plusieurs combattants entrent en lice qui, " comme la loi l'ordonnait ", doivent inscrire leur " devise » en cachant leur "visage " et leur «nom ». Le récit n'en caractérise que trois : « un seigneur très riche, nommé Itobad, fort vain, peu courageux, très maladroit, et sans esprit " porte "une armure d'or émaillée de vert, un panache vert, une lance ornée de rubans verts »; le prince Otame, qui porte "des armes bleues et or, avec un panache de même "; enfin Zadig, dont les armes sont «blanches ». Le premier ayant aussitôt été défait - et ridiculisé -, «tous les vœux se partageaient entre le cavalier bleu et le cavalier blanc » et, à l'issue des joutes entre le "prince bleu » et le « cavalier blanc », c'est le « chevalier blanc » qui l'emporte : «On reconduisit le chevalier bleu et le chevalier blanc chacun à leur loge, ainsi que tous les autres, selon ce qui était porté par la loi.» Mais la loi du récit veut aussi que, pendant la nuit, Itobad, au nom programmatique (is too bad?), prenne " les armes blanches de Zadig avec sa devise » et mette « son armure verte à la place » : il est aussitôt "proclamé ». On voit comment la narration thématise à la fois le mode de fonctionnement du code de reconnaissance et les limites de ce code : si Zadig, auquel il " échappa [...] de murmurer contre la Providence ", «fut tenté de croire que tout était gouverné par une destinée cruelle qui opprimait les bons et qui faisait prospérer les chevaliers verts $»^{21}$, on peut aussi voir à l'œuvre une providence d'auteur qui, jouant avec les codes narratifs, en l'occurrence ce que les formalistes russes ont désigné comme la "reconnaissance du faux héros ${ }^{22}$ ", relance ainsi une intrigue dont il diffère le dénouement. 
8 Voltaire avait précédemment mis en œuvre un jeu équivalent sur les couleurs emblématiques, plus exactement sur la signification de la rhétorique des couleurs - couleurs apparemment arbitraires mais dont l'interprétation révèle la signification secrète. C'est alors le roi Moabdar, en proie à une jalousie qui n'est pas sans fondement, qui joue le rôle de l'herméneute. Après avoir décrypté la physionomie de la reine lorsqu'il est question de Zadig (la "rougeur» dont "son front se couvrait», son attitude «tantôt si animée, tantôt si interdite", la «rêverie si profonde" qui «s'emparait d'elle»), «il crut tout ce qu'il voyait, et imagina tout ce qu'il ne voyait point » :

Il remarqua surtout que les babouches de sa femme étaient bleues, et que les babouches de Zadig étaient bleues, que les rubans de sa femme étaient jaunes, et que le bonnet de Zadig était jaune : c'étaient là de terribles indices pour un prince délicat ${ }^{23}$.

Circonstance aggravante, alors que, dans un épisode antérieur, Zadig avait refusé de rattacher au genou de la femme d'Arimaze, « l'envieux » qui se voue à la perte de Zadig, la jarretière qu'elle avait opportunément laissé tomber ${ }^{24}$, «l'envieux engagea l'envieuse à envoyer au roi sa jarretière, qui ressemblait à celle de la reine » : «Par surcroît de malheur, cette jarretière était bleue.» Devant un faisceau d'indices aussi confondant, Moabdar « résolut [...] d'empoisonner la reine, et de faire mourir Zadig par le cordeau, au point du jour $\aleph^{25}$. À cette première séquence de décodage des signes - la coïncidence fait sens et accrédite l'existence d'un langage des couleurs - succède une séquence d'encodage mettant en jeu ce langage : l'ordre d'exécution donné à « un impitoyable eunuque » est surpris par « un petit nain » qui malheureusement «était muet » mais « n'était pas sourd », et surtout « était très attaché à la reine et à Zadig » qu'il s'efforce d'avertir avec les moyens dont il dispose. Certes, «il ne savait pas écrire ; mais il avait appris à peindre, et savait surtout faire ressembler » :

Il passa une partie de la nuit à crayonner ce qu'il voulait faire entendre à la reine. Son dessin représentait le roi agité de fureur, dans un coin du tableau, donnant des ordres à son eunuque ; un cordeau bleu et un vase sur la table, avec des jarretières bleues et des rubans jaunes ; la reine, dans le milieu du tableau, expirante entre les bras de ses femmes, et Zadig étranglé à ses pieds. L'horizon représentait un soleil levant, pour marquer que cette horrible exécution devait se faire aux premiers rayons de l'aurore.

Les signes sont parfaitement décodés en retour par Astarté qui ordonne aussitôt à Zadig de fuir, écrit-elle, « au nom de notre amour et de mes rubans jaunes ${ }^{26} »$. Et, au cours des épisodes suivants, Zadig de se remémorer qu'il « a été sur le point d'être étranglé parce que la reine avait des rubans jaunes ${ }^{27} "$, Astarté de rappeler, au moment de leurs retrouvailles, que cette nuit-là un officier s'était rendu chez lui « avec un lacet de soie bleue $^{28}$ ». Au vu de ces jeux d'encodage et de décodage minutieux, on peut néanmoins s'étonner de l'inadvertance du conteur lorsque, entre-temps, il rapporte les réflexions de son personnage "sur le sort qui s'obstinait à se jouer de lui et à le persécuter »: «[...] prêt à être étranglé parce que la reine avait des babouches de la couleur de mon bonnet $^{29}$ !»

9 Ce raté mis à part, le travail, dans l'épisode de «la jalousie », sur la rhétorique des couleurs a pour bénéfice narratif de permettre aux lecteurs et lectrices d'anticiper sur la crise à venir mais aussi, dans un conte fortement métapoétique, de mettre en place ce qui a été désigné, à la suite de Carlo Ginzburg, comme le " paradigme indiciaire ${ }^{30}$ »: le mécanisme de décryptage des indices, dont le mode de fonctionnement est exposé dans l'épisode qui met en scène Zadig lorsqu'il transforme en signes les traces laissées 
par la chienne de la reine et le cheval du roi ${ }^{31}$, participe d'un jeu avec les lecteurs et lectrices à leur tour mis en situation d'anticiper sur la suite du récit à partir des indices qui leur sont fournis, en particulier sous la forme de notations de couleurs. On s'en tiendra à quelques exemples tirés d'autres contes. Parmi le bestiaire étrange de Candide $^{32}$, on se souvient de la découverte faite en Eldorado de "gros moutons rouges qui surpassaient en vitesse les plus beaux chevaux d'Andalousie, de Tétuan et de Méquinez ${ }^{33}$ ", qui servent de moyens de locomotion: "deux grands moutons rouges sellés et bridés " sont donnés à Candide et Cacambo "pour leur servir de monture " lorsqu'ils décident de sortir du pays, vingt autres étant "chargés de vivres ", trente portant « des présents de ce que le pays a de plus curieux » et " cinquante chargés d'or, de pierreries et de diamants $\aleph^{34}$. On se souvient aussi que l'équipage ne tarde pas à diminuer, jusqu'à ce bilan: Candide a "perdu cent gros moutons rouges chargés des plus grands trésors de la terre", les deux derniers ${ }^{35}$ ayant disparu à cause de «la friponnerie du patron hollandaii ${ }^{36} »$ qui, après avoir été grassement payé pour conduire Candide à Venise, s'est enfui en mer avec les moutons et leur chargement, laissant le voyageur sur le rivage. Alors que le valet métis est parti racheter Cunégonde, Candide recrute un nouveau compagnon et s'embarque pour Bordeaux. Des coups de canon retentissent, deux vaisseaux combattent sur mer, l'un est coulé à fond, Candide et Martin assistent à la noyade de l'équipage et Candide convient « qu'il y a quelque chose de diabolique dans cette affaire »: «En parlant ainsi, il aperçut je ne sais quoi d'un rouge éclatant qui nageait auprès de son vaisseau. » L'ignorance affichée d'un conteur qui rappelle discrètement sa présence est censée susciter la sagacité des lecteurs et lectrices, qui ne tardent pas du reste à connaître la solution de l'énigme : «On détacha la chaloupe pour voir ce que ce pouvait être : c'était un de ses moutons. " Et Candide d'en tirer bon augure: s'il a "retrouvé » son mouton, il pourra bien «retrouver Cunégonde $»^{37}$.

Le même procédé est repris au moment des retrouvailles avec Cacambo qui devait ramener Cunégonde à Venise et dont l'absence au rendez-vous est rappelée avec insistance au début de l'épisode («nulles nouvelles de Cacambo»), ce qui plonge Candide dans « une mélancolie noire $»^{38}$, et dramatisée plus loin («Cependant les jours, les semaines s'écoulaient; Cacambo ne revenait point $\left.{ }^{39} »\right)$. Le chapitre suivant commence ainsi :

Un soir que Candide, suivi de Martin, allait se mettre à table avec les étrangers qui logeaient dans la même hôtellerie, un homme à visage couleur de suie l'aborda parderrière, et, le prenant par le bras, lui dit: «Soyez prêt à partir avec nous, n'y manquez pas. » Il se retourne, et voit Cacambo ${ }^{40}$.

Le schéma est encore une fois exploité au cours de l'épisode du baptême de l'Ingénu qui, alors que toute la compagnie basse-bretonne est conviée à la cérémonie, avec l'évêque de Saint-Malo et son "pompeux équipage», disparaît ("on ne le trouva point »; " point de nouvelles du Huron »), suscitant le désespoir du prieur et de l'abbé de Saint-Yves, les pleurs de $\mathrm{M}^{\text {lle }}$ de Kerkabon et les soupirs de $\mathrm{M}^{\text {lle }}$ de Saint-Yves :

Elles se promenaient tristement le long des saules et des roseaux qui bordent la petite rivière de Rance, lorsqu'elles aperçurent au milieu de la rivière une grande figure assez blanche, les deux mains croisées sur la poitrine. Elles jetèrent un grand cri et se détournèrent. Mais la curiosité l'emportant bientôt sur toute autre considération, elles se coulèrent doucement entre les roseaux, et quand elles furent bien sûres de n'être point vues, elles voulurent voir de quoi il s'agissait.

L'effet de chapitrage ne permet pas de mesurer la durée de la contemplation de cette "grande figure assez blanche", qui n'est identifiée, au début du chapitre suivant, 
qu'avec l'entrée en scène du prieur et de l'abbé : ils « demandèrent à l'Ingénu ce qu'il faisait là ${ }^{41} »$.

11 Dans Candide, les indices permettant la reconnaissance du frère du baron de Thunderten-tronck, dont la mort avait successivement été attestée par Pangloss («traité comme sa sœur ", qui " a été éventrée par des soldats bulgares, après avoir été violée autant qu'on peut l'être $»^{42}$ ) et par Cunégonde elle-même ( ils égorgèrent mon père et mon frèr $\left.\mathrm{e}^{43} »\right)$, sont encore plus subtils : parvenus au Paraguay, Candide et Cacambo attendent le révérend père qui commande la réduction jésuite et voient apparaître « un très beau jeune homme, le visage plein, assez blanc, haut en couleur [...] l'air fier, mais d'une fierté qui n'était ni celle d'un Espagnol ni celle d'un jésuite ». Avant même la scène de retrouvaille, traitée sur le mode parodique ("Quel miracle!», "Serait-ce vous? », «Cela n'est pas possible »), cet indice suggérant un personnage déplacé dans le contexte jésuite de la colonie espagnole est précédé d'un autre, pour qui conserve la mémoire du texte: non seulement les références antérieures suggéraient une communauté de destin entre le frère et la sœur (si la sœur a survécu, pourquoi pas le frère ?), mais le portrait du révérend père, "haut en couleur ", fait directement référence à celui de Cunégonde, brossé, au début du conte : «Cunégonde, âgée de dixsept ans, était haute en couleur, fraîche, grasse, appétissante ${ }^{44}$.»

Voltaire effectue ainsi un usage rhétorique des couleurs qui participe de la dynamique narrative des récits : le processus d'identification des personnages repose en particulier sur la mise en place d'un " paradigme indiciaire » sollicitant l'activité de ses lecteurs et lectrices. Si lorsqu'il s'agit de personnages secondaires plus ou moins prototypiques, la simple mention de la couleur du vêtement constitue un indice économique suffisant, on va voir que les textes exploitent davantage encore - et occasionnellement problématisent - une symbolique des couleurs dans la caractérisation des personnages de premier plan.

\section{La peau de Candide}

Les personnages sont en effet caractérisés de telle sorte que se trouvent mis en question, à travers la valeur esthétique des couleurs, les canons de la beauté et de la laideur ${ }^{45}$. Les contes de Voltaire reprennent ainsi les données fondamentales de la beauté féminine : la blancheur de la peau rehaussée par la coloration vive de la bouche et du teint. Dans Zadig, le récit de l'entreprise de séduction du chef des prêtres des étoiles souligne « la forme admirable » et la «blancheur éblouissante » des bras de la jeune veuve Almona, prélude à une scène de strip-tease relatée avec distance à grand renfort de «style oriental ${ }^{46} »$ :

Le pontife [...] jura qu'il n'avait pas vu de sa vie de si beaux bras. « Hélas! lui dit la veuve, les bras peuvent être un peu moins mal que le reste; mais vous m'avouerez que la gorge n'était pas digne de mes attentions. » Alors elle laissa voir le sein le plus charmant que la nature eût formé. Un bouton de rose sur une pomme d'ivoire n'eût paru auprès que de la garance sur du buis, et les agneaux sortant du lavoir auraient semblé d'un jaune brun. Cette gorge, ses grands yeux noirs qui languissaient en brillant doucement d'un feu tendre, ses joues animées de la plus belle pourpre mêlée au blanc du lait le plus pur, son nez, qui n'était pas comme la tour du mont Liban, ses lèvres, qui étaient comme deux bordures de corail renfermant les plus belles perles de la mer d'Arabie, tout cela ensemble fit croire au vieillard qu'il avait vingt ans ${ }^{47}$. 
Tels sont aussi les traits caractéristiques de la femme du pêcheur («l'éclat de la pourpre de Tyr n'était pas plus brillant que l'incarnat qui animait cette blancheur $\left.{ }^{48} »\right)$, tout comme, dans l'Histoire de Jenni (1775), de doña Boca Vermeja, au nom programmatique, que délaisse cependant Jenni au profit de $\mathrm{M}^{\text {me }}$ Clive-Hart - dont le nom est également évocateur -, « parce que la Clive-Hart avait une nuance ou deux de blancheur et d'incarnat au-dessus de la pauvre Boca Vermeja ${ }^{49}$ ".

Ces portraits dont les couleurs récurrentes accusent la dimension stéréotypée ${ }^{50}$ laissent cependant place à des nuances. Ainsi, dans La Princesse de Babylone, de Formosante, la bien nommée, dont la «belle bouche » est comparée au «bec [...] couleur de rose » du phénix ${ }^{51}$, mais qui, aux yeux des Espagnols, «n'est pas si belle » car "il n'y a de beau que les teints basanés » : "[...] elle étale une gorge d'albâtre qui est la chose du monde la plus dégoûtante, et qu'on ne connaît point dans [leurs] climats. $»^{52}$ Confirmation, si nécessaire, que "le beau est souvent très relatif», comme l'écrivait Voltaire dans l'article «Beau» (1764) du Dictionnaire philosophique ${ }^{53}$. À côté des blondes, les textes comptent ainsi un certain nombre de brunes, à l'instar de la «jolie brune ${ }^{54}$ » avec laquelle, à Paris, dans La Princesse de Babylone, Amazan commet sa seule infidélité, et déjà de la maîtresse du Saturnien (« une jolie petite brune ${ }^{55}$ ») dans Micromégas, de la femme de Colin ${ }^{56}$, ou encore de la femme de chambre de la baronne de Thunder-tentronck, à laquelle Pangloss donne "une leçon de physique expérimentale»: une " petite brune très jolie et très docile ${ }^{57}$ ». La couleur des yeux est aussi objet de débats dans un épisode de Zadig retrouvé dans les papiers de Voltaire après sa mort, significativement intitulé "Les yeux bleus ", relatant l'origine de l'« ancienne loi qui défendait aux rois d'aimer une de ces femmes que les Grecs ont appelées depuis boopies » : épris de « la belle Falide », le roi Nabussan « aimait deux grands yeux bleus ", «les bossus, les financiers, les bonzes et les brunes remplirent le royaume de leurs plaintes » et, lorsque Zadig souffle au roi le moyen d'anéantir la résistance des bonzes, «les bonzes et les brunes jur[ent] sa perte $»^{58}$.

Au-delà des effets de relativisme, le rappel et le traitement des topiques conventionnels de la beauté féminine permettent d'introduire dans les textes diverses formes d'écarts que les notations de couleurs révèlent et soulignent. Celui, d'abord, lié au vieillissement, qu'illustre le personnage de la vieille dans Candide qui, racontant son «histoire », déclare: "Je n'ai pas eu toujours les yeux éraillés et bordés d'écarlate; mon nez n'a pas toujours touché mon menton [...]. » Peu après, son autoportrait, à l'âge de quatorze ans, malgré la chute ironique, reprend les canons de beauté déjà évoqués et forme un contraste saisissant :

J'inspirais déjà de l'amour, ma gorge se formait; et quelle gorge! blanche, ferme, taillée comme celle de la Vénus de Médicis; et quels yeux ! quelles paupières ! quels sourcils noirs! quelles flammes brillaient dans mes deux prunelles, et effaçaient la scintillation des étoiles, comme me le disaient les poètes du quartier ${ }^{59}$.

Ce contraste n'a d'égal que celui qui saisit Candide lorsque, à la fin du conte, il retrouve enfin celle qu'il n'a cessé de désigner comme "la belle Cunégonde", l'expression, devenue presque une épithète homérique, étant ici reprise dans un ultime effet d'ironie cinglante : «Le tendre amant Candide, en voyant sa belle Cunégonde rembrunie, les yeux éraillés, la gorge sèche, les joues ridées, les bras rouges et écaillés, recula de trois pas, saisi d'horreur $[. . .]^{60}$. » Ainsi se construit aussi une parenté de destin qui fait de la vieille le double de la fille du baron ${ }^{61} \ldots$

Dans Le Crocheteur borgne, c'est plutôt de disconvenance sociale qu'il est question: le premier en date des contes de Voltaire, composé vers 1715 , met en scène l'union 
quelque peu scabreuse de la princesse Mélinade - qui ressemble à s'y méprendre à la duchesse du Maine, à la cour de laquelle le texte a été créé - et d'un portefaix disgracié. Le contraste, présenté en point de vue interne, thématisé par la " comparaison » que - quoique borgne - le crocheteur a « faite en chemin entre lui et sa maitresse", est souligné par une opposition entre le blanc et le blond qui caractérisent la première ${ }^{62}$, le brun et le noir qui sont les couleurs dominantes du second:

Elle avait une robe d'une légère étoffe d'argent, semée de guirlandes de fleurs, qui faisait briller la beauté de sa taille; et lui avait un sarrau brun taché en mille endroits, troué et rapiécé [...]. Il avait comparé ses mains nerveuses et couvertes de durillons avec deux petites mains plus blanches et plus délicates que les lis. Enfin il avait vu les beaux cheveux blonds de Mélinade, qui paraissaient à travers un léger voile de gaze, relevés les uns en tresse et les autres en boucles; et il n'avait à mettre à côté de cela que des crins noirs hérissés, crépus, et n'ayant pour tout ornement qu'un turban déchiré63.

17 Le scandale que suscite l'union de deux personnages si dissemblables (« il fut brutal et heureux $\left.{ }^{64} »\right)$ est cependant doublement compensé. D'une part, par la transfiguration du crocheteur en « un jeune homme d'une taille noble », ainsi décrit à travers le regard de Mélinade à son réveil :

Il avait des joues de rose, des lèvres de corail ; ses grands yeux, tendres et vifs tout à la fois, exprimaient et inspiraient la volupté ; son carquois d'or, orné de pierreries, était suspendu à ses épaules, et le plaisir faisait seul sonner ses flèches; sa longue chevelure, retenue par une attache de diamants, flottait librement sur ses reins, et une étoffe transparente, brodée de perles, lui servait d'habillement et ne cachait rien de la beauté de son corps ${ }^{65}$.

18 D'autre part, par la structure même du conte qui, avec le réveil brutal du crocheteur, est renvoyé à un songe aviné.

19 À l'opposé chronologique dans la production voltairienne, c'est une semblable métamorphose qui rend acceptable l'attirance encore plus scabreuse de la princesse Amaside pour un «taureau [...] blanc, fait au tour, potelé, léger même, ce qui est bien rare ", quels que soient les précédents mythologiques ${ }^{66}$. Le sortilège étant dissipé, Nabuchodonosor, entre-temps promu successeur du dieu Apis, reprend forme humaine :

On vit d'un coup le dieu perdre ses deux jambes de derrière; ses deux jambes de devant se changèrent en deux jambes humaines; deux beaux bras charnus, musculeux et blancs sortirent de ses épaules; son mufle de taureau fit place au visage d'un héros charmant; il redevint le plus bel homme de la terre $[. . .]^{67}$.

Reste tout de même le titre du chapitre, qui annonce assez crûment que son objet est de raconter « comment la princesse épousa son bœuf »...

Si en dehors de la blancheur ${ }^{68}$ le portrait de Nabuchodonosor présente tous les codes de la masculinité, tel n'est pas le cas du crocheteur, du moins tel qu'il est présenté du point de vue de Mélinade, avec ses "joues de rose", ses "lèvres de corail », pour ne rien dire de sa "longue chevelure $»^{69}$. Le cas n'est pas isolé dans les contes, qui présentent des beautés plus ou moins androgynes: dans l'Histoire de Jenni, $\mathrm{M}^{\mathrm{me}}$ CliveHart, on l'a vu, surpasse Boca Vermeja en «blancheur » et en « incarnat », mais elle est présentée, peu avant, comme "très effrontée, très emportée, très masculine, très méchante $»^{70}$. Dans La Princesse de Babylone, Amazan a «le visage d'Adonis sur le corps d'Hercule » et allie " la majesté avec les grâces ", au point que, lorsqu'il entre en scène, «ses sourcils noirs et ses longs cheveux blonds, mélange de beauté inconnue à 
Babylone $^{71}$ ", charment l'assemblée. Tel paraît encore être l'Ingénu, baptisé Hercule, qui dès son apparition ravit $\mathrm{M}^{\text {lle }}$ de Kerkabon : «Ce grand garçon-là a un teint de lis et de rose ! qu'il a une belle peau pour un Huron ${ }^{72}$ ! » Préjugé de race mis à part suggérant discrètement l'étroitesse d'esprit des Bas-Bretons, c'est encore ce qui retient l'attention de Cunégonde : lorsqu'elle évoque le capitaine qui l'a tirée des mains du «brutal » en train de la violer, elle ne peut nier « qu'il ne fût très bien fait, et qu'il n'eût la peau blanche et douce » quoique, précise-t-elle plus loin à Candide, elle avoue " avec vérité ", après l'avoir vu « dépouillé tout nu » se faire fesser par l'Inquisition, que sa " peau est encore plus blanche et d'un incarnat plus parfait que celle de [s]on capitaine des Bulgares $»^{73}$. On se souvient encore du portrait du frère de Cunégonde lors de sa réapparition en révérend père commandant au Paraguay, qu'il est temps de compléter : « un très beau jeune homme, le visage plein, assez blanc, haut en couleur, le sourcil relevé, l'œil vif, l'oreille rouge, les lèvres vermeilles, l'air fier ${ }^{74}$ ». Mais le trouble dans le genre est à son comble dans l'évocation, par doña Las Nalgas, rédactrice de l'« Aventure » de Jenni, du « jeune English » au sortir du bain :

[...] il ôta un petit bonnet sous lequel étaient renoués ses cheveux blonds, qui descendirent en grosses boucles sur la plus belle chute de reins que j'ai vue de ma vie ; ses bras, ses cuisses, ses jambes, me parurent d'un charnu, d'un fini, d'une élégance qui approche à mon gré l'Apollon du Belvédère de Rome $[. . .]^{75}$.

Outre qu'elle questionne la définition binaire des identités de genre ${ }^{76}$, la réduction au semblable dénoue les tensions engendrées par les situations de disconvenance, qui sont autant d'infractions à la loi du même, autrement dit du stéréotype. Les topiques définissant les canons de la beauté, en particulier féminine, n'en font pas moins l'objet d'un traitement à distance qui a pour incidence de souligner l'artifice qui préside au recours assumé à ces topiques (blancheur, blondeur). Ces représentations conventionnelles sont aussi à l'occasion questionnées lorsque la mise en scène de beautés détonnantes conduit au relativisme en mettant en évidence la diversité des êtres. La présentation de cette diversité, en particulier envisagée du point de vue de la couleur de la peau, est en outre sous-tendue par une réflexion philosophique qui comporte plusieurs volets.

\section{Des personnages de toutes couleurs}

Si la blancheur reste, moyennant quelques nuances, symboliquement attachée à la beauté, elle est aussi, dans d'autres contextes, un marqueur de sagesse à l'instar, dans Zadig, de «la barbe blanche et vénérable » de l'ermite " qui lui descendait jusqu'à la ceinture » et qui trouve son pendant, au terme de la métamorphose du personnage en ange Jesrad, dans les "quatre belles ailes" qui couvrent "un corps majestueux et resplendissant de lumière $»^{77}$. L'association est encore plus explicite, dans Le Blanc et le Noir, à propos de l'un des « deux favoris » de Rustan, « beau, bien fait, blanc comme une Circassienne, doux et serviable comme un Arménien, sage comme un Guèbre ", désigné comme «le sage Topaze $»^{78}$. L'autre, significativement nommé Ébène, "un nègre fort joli » désigné comme son « cher nègre $e^{79}$ ", constitue le double inversé du précédent, le contraste entre leurs couleurs étant confirmé par leurs réactions opposées face au projet de Rustan de quitter Candahar pour retrouver la princesse de Cachemire dont il est épris : Topaze s'oppose à ce voyage alors qu'Ébène l'encourage. En termes actantiels, le blanc fait figure d'opposant, le noir d'adjuvant, ce qui se vérifie lors de chacune des péripéties que doit affronter Rustan jusqu'à son arrivée dans la province de Cachemire, 
qui correspond au moment de sa mort. Une double métamorphose est alors décrite («quatre ailes blanches couvrirent le corps de Topaze, et quatre ailes noires celui d'Ébène »), et alors que, face à l'étonnement du jeune homme de Candahar, les deux personnages interprètent le sens de cet événement merveilleux, l'opposition des couleurs se charge de valeurs axiologiques: l'un et l'autre sont ses «deux génies »; Topaze, son «bon génie», Ébène, son "mauvais génie " ${ }^{80}$. Dans un effet de renversement, cette axiologisation rétablit la signification polarisée des figures actantielles : le terme du voyage étant la mort de Rustan, l'opposant (blanc et bon) redevient l'adjuvant, et vice versa.

Là ne s'arrête pourtant pas le jeu des renversements, corrélés à la mise au jour de principes d'intelligibilité, qui s'enchaînent de manière vertigineuse dans le conte. D'abord, alors que Rustan déclare qu'« il y a quelque chose là-dessous " qu'il «ne compren[d] pas »- perplexité que partage « le pauvre bon génie »-, « il se réveille en sursaut, tout en sueur, tout égaré »; « son valet de chambre Topaze accourt en bonnet de nuit » et Ébène "ronfle » à l'étage ${ }^{81}$ : ce qui précède se trouve brusquement renvoyé à un rêve, les manifestations du merveilleux sont rationalisées et le mystère quelque peu dissipé. Les explications fournies par Topaze (« vous avez rêvé tout cela [...]. Dieu a voulu que cette file d'idées vous ait passé par la tête, pour vous donner apparemment quelque instruction dont vous ferez votre profit »), puis ses réflexions sur la marche du temps suscitent cependant de nouvelles perplexités: "Je n'y entends rien, dit Rustan $^{82}$.» Une nouvelle perspective d'explication s'offre alors, suggère Topaze : «[...] j'ai un perroquet qui vous le fera aisément comprendre. Il est né quelque temps avant le déluge ; il a beaucoup vu; cependant il n'a encore qu'un an et demi [...]. » Mais au moment où la lumière s'apprête à surgir («On lui amena le perroquet, lequel parla ainsi »), une note de l'éditeur achève inopinément le récit avec désinvolture et relance les perplexités non plus du personnage mais des lecteurs et lectrices :

N. B. - Mlle Catherine Vadé n'a jamais pu trouver l'histoire du perroquet dans le portefeuille

de feu son cousin Antoine Vadé, auteur de ce conte. C'est grand dommage, vu le temps auquel vivait ce perroquet ${ }^{83}$.

Un tel dénouement concourt à la mise en crise du conte dans sa capacité à rendre intelligible le réel : alors que, dans Zadig, le perroquet du roi permettait de redresser le sens du poème de Zadig rendu trompeur par sa version tronquée ${ }^{84}$, ici le perroquet, conteur et herméneute, est tout simplement absent. Ne subsistent que des discours sur le réel, dont le conte met en scène de manière comique le caractère ambivalent et réversible, et qui, avec la thématisation de l'incompréhension à répétition, n'ont pas de pertinence avérée. Si les insuffisances des discours métaphysiques sont ici pointées en général, il en va de même, en particulier, de la grille de lecture manichéenne - le blanc, le bon ; le noir, le mal -, inopérante à rendre compte de l'origine du bien et du mal.

Dans d'autres contes, l'évocation de la couleur de la peau des personnages ${ }^{85}$ est le support d'un discours anthropologique. On retrouve ici l'idée d'une diversité des êtres humains, déjà rencontrée à propos de la question de la beauté, qu'expriment et rendent visible les couleurs. On pense, dans La Princesse de Babylone, au « prince noir » d'Éthiopie encore désigné, plus loin, comme le "monarque noir », qui se distingue du "vieux roi tanné » de la Bétique ${ }^{86}$. Il est aussi question, dans la "Lettre à l'homme aux quarante écus ", de la peau des Caraïbes, dans le contexte d'une polémique avec le libraire qui imprime le Journal économique : 
Il me nia impudemment que les Caraïbes fussent nés rouges. Je fus obligé de lui envoyer une attestation d'un ancien procureur du roi de la Guadeloupe, comme quoi Dieu a fait les Caraïbes rouges, ainsi que les nègres noirs ${ }^{87}$.

Dans Candide, la vieille évoque aussi les «cinquante guerres civiles » qui ravagent le Maroc lorsque, devenue esclave, elle constate le «carnage continuel» qui affecte «toute l'étendue de l'empire »: « noirs contre noirs, [...] noirs contre basanés, [...] basanés contre basanés, [...] mulâtres contre mulâtres $»^{88}$. Le personnage de Cacambo, dont on a déjà évoqué la reconnaissance par le truchement de son "visage couleur de suie », est encore emblématique d'un métissage : « C'était un quart d'Espagnol, né d'un métis dans le Tucuman $[\ldots]^{89}$.

Ces marqueurs de la diversité sont toutefois occasionnellement traduits en terme de différence irréductible. Dans l'Histoire de Jenni, le discours que Freind oppose à celui, ouvertement athée, de Birton fait référence à une multitude de peuples, Esquimaux, Islandais, Lapons, Ostiakes, Samoyèdes, et il ajoute :

Il est clair que Dieu a fait chaque espèce d'animaux et de végétaux pour la place dans laquelle ils se perpétuent. Les nègres, cette espèce d'hommes si différente de la nôtre, sont tellement nés pour leur patrie que des milliers de ces animaux noirs se sont donné la mort quand notre barbare avarice les a transportés ailleurs ${ }^{90}$.

La dernière étape du voyage de Scarmentado l'amène en Afrique, à son corps défendant puisque son vaisseau est "pris par des corsaires nègres". Réfutant toute idée de violation des « lois des nations ", le « capitaine nègre » argue d'une différence radicale de constitution physique pour naturaliser les rapports de pouvoir :

Vous avez le nez long, et nous l'avons plat; vos cheveux sont tout droits, et notre laine est frisée; vous avez la peau couleur de cendre, et nous de couleur d'ébène ; par conséquent nous devons, par les lois sacrées de la nature, être toujours ennemis.

Par la suite, le corsaire invoque pourtant les traitements infligés aux gens de son espèce («Vous nous achetez aux foires de la côte de Guinée comme des bêtes de somme, pour nous faire travailler à je ne sais quel emploi aussi pénible que ridicule ») avant de conclure, selon une logique non plus fondée sur l'argument de nature mais bien sur celui du talion, à l'exercice légitime de la loi du plus fort : «[...] aussi, quand nous vous rencontrons et que nous sommes les plus forts, nous vous faisons esclaves, nous vous faisons labourer nos champs, ou nous vous coupons le nez et les oreilles ${ }^{91}$. $\gg$ Ici comme dans le discours de Freind, qui dénonce "notre barbare avarice ", les considérations anthropologiques sont mises au service d'une critique virulente de la brutalité du commerce triangulaire mettant en jeu une "marchandise humaine ${ }^{92}$ ", dans une économie en quelque sorte mondialisée.

"C'est à ce prix que vous mangez du sucre en Europe ", constate à son tour le nègre de Surinam, qui souligne également un autre scandale lorsqu'il rappelle ce que disent et répètent, «tous les dimanches », les « fétiches hollandais » qui l'ont « converti » : « [...] nous sommes tous enfants d'Adam, blancs et noirs. » La question de la différence, ici niée - mais alors il faut en conclure, de manière très conséquente, « qu'on ne peut pas en user avec ses parents d'une manière plus horrible $»^{93}$ - est ailleurs affirmée d'une manière aussi polémique que provocatrice. « Nos sages ont dit que l'homme est l'image de Dieu : voilà une plaisante image de l'Être éternel qu'un nez noir épaté, avec peu ou point d'intelligence ", déclare Amabed, qui se livrait déjà, dans une lettre antérieure, à des réflexions du même acabit sur les Hottentots :

Plus je réfléchis sur la couleur de ces peuples, sur le glossement dont ils se servent pour se faire entendre, au lieu d'un langage articulé, sur leur figure, sur le tablier de 
leurs dames, plus je suis convaincu que cette race ne peut avoir la même origine que nous.

Notre aumônier prétend que les Hottentots, les nègres et les Portugais descendent $\mathrm{du}$ même père. Cette idée est bien ridicule. J'aimerais autant qu'on me dît que les poules, les arbres et l'herbe de ce pays-là viennent des poules, des arbres et de l'herbe de Bénarès ou de Pékin ${ }^{94}$.

Au-delà de considérations que l'on peut à bon droit juger racistes, ce qui est ici en jeu, comme l'ont montré les analyses de Michèle Duchet et de José-Michel Moureaux ${ }^{95}$, c'est bien l'affirmation d'une thèse polygéniste mise au service de la polémique antichrétienne, plus précisément anti-biblique, le livre de la Genèse étant ouvertement en ligne de mire.

Les couleurs servent encore de support à l'intrusion, dans le récit, de la satire de l'institution ecclésiastique. On a évoqué, au début de cette étude, la valeur catégorisante, en contexte oriental, des mages "blancs ", " noirs ", voire " de toutes couleurs $»^{96}$, ou, dans une moins exotique Europe, le "prédicant à manteau noir» de l'Histoire des voyages de Scarmentado, auquel fait encore écho le "monsieur à manteau noir » qui rudoie Candide en Hollande ${ }^{97}$. Dans les contes, la couleur noire désigne aussi, de manière plus spécifique, les acteurs de l'Inquisition : ainsi du «petit homme noir familier de l'Inquisition ${ }^{98}{ }$ qui surprend le discours que Pangloss adresse aux habitants de Lisbonne après le tremblement de terre, ce qui lui vaut, ainsi qu'à Candide, emprisonnement et autodafé. Ainsi encore, dans l'Histoire de Jenni, du « spectre noir en surplis » qui sort du soupirail d'une cave, bientôt identifié comme "don Jeronimo Bueno Caracucarador, inquisiteur pour la foi $»^{99}$, ou des " cinq personnages vêtus de robes noires avec une chemise par-dessus leur robe, et deux longs pendants d'étoffe bigarrée par-dessus leur chemise » que voit apparaître Adaté dans la "prison de l'Inquisition", l'un de ces "monstres noirs et blancs" la déclarant peu après «Apostata! » ${ }^{100}$. Lors du séjour d'Amazan dans la capitale des Gaules, ce sont encore les couleurs qui permettent de distinguer les jésuites - des « ex-druides, vêtus de noir »et les jansénistes - «des boucs vêtus de gris $»^{101}$.

Mais c'est sans conteste la cour de Rome qui offre le spectacle le plus coloré. Amazan apprend ainsi que le « roi des rois » - ou plutôt le «serviteur des serviteurs » - dispose de "quatre à cinq cent mille prophètes divins distribués dans les autres pays »: des " prophètes de toutes couleurs » qui «sont, comme de raison, nourris aux dépens des peuples ». Des couleurs plus spécifiquement signifiantes servent surtout à marquer les différences au sein de la hiérarchie ecclésiastique, quoique les uns et les autres - trait de satire collatéral - s'accordent pour n'être pas insensibles au charme du «bel Amazan » :

Il vit bientôt défiler toute la cour du maître du monde: elle était composée de graves personnages, les uns en robes rouges, les autres en violet; presque tous regardaient le bel Amazan en adoucissant les yeux ; ils lui faisaient des révérences, et se disaient l'un à l'autre: San Martino, che bel ragazzo! San Pancratio, che bel fanciullo ${ }^{102}$ !

La satire se prolonge par la suite lorsque Amazan, après avoir appris que, s'il veut rencontrer le «Vieux des sept montagnes » il convient de faire trois génuflexions et de lui baiser les pieds, est en butte aux « propositions encore plus étranges » des « seigneurs les plus importants de la ville », les personnages étant désormais désignés de manière plus économique, par réduction métonymique et substantivation : " un violet », "le violet », « deux ou trois des plus déterminés violets » qu'il finit par jeter «par les 
fenêtres, sans croire faire un grand sacrifice à la belle Formosante ». Le séjour romain tourne court :

Il quitta au plus vite cette ville des maitres du monde, où il fallait baiser un vieillard

à l'orteil, comme si sa joue était à son pied, et où l'on n'abordait les jeunes gens qu'avec des cérémonies encore plus bizarres ${ }^{103}$.

Le décryptage fondé sur une phénoménologie des couleurs se double ici, par la mise en place du dispositif du regard décalé porté par l'étranger sur les us et coutumes européens, des vertus critiques de l'« estrangement ", selon la notion élaborée par Carlo Ginzburg ${ }^{104}$. La même expérience est donnée à lire, dans le même contexte, dans les lettres d'Adaté et d'Amabed. La première décrit à l'intention de Shastasid, grand brame de Maduré, « l'obispo de Goa »:

Un obispo est à peu près parmi ces barbares ce que tu es chez les enfants de Brahma ; c'est un intendant de leur religion; il est vêtu de violet, et il porte aux mains des souliers violets ${ }^{105}$.

Amabed, de son côté, évoque les «hommes vêtus de rouge et inspirés » qui « élurent l'homme infaillible » censé le juger ainsi qu'Adaté « en qualité d'apostata » ${ }^{106}$. Mais, dans ce conte, la satire prend une forme moins virulente, du moins plus ambiguë: la dernière mention de « deux jeunes gens vêtus de rouge de la tête aux pieds » intervient dans un épisode où Amabed et Adaté se voient offrir un «excellent repas " ${ }^{107}$ chez le « monsignor violet » qui « a pris en amitié » Amabed $^{108}$. Le conte s'interrompt ici encore de manière inattendue: "Ici finit le manuscrit des lettres d'Amabed. On a cherché dans toutes les bibliothèques de Maduré et de Bénarès la suite de ces lettres. Il est sûr qu'elle n'existe pas $^{109}$. " Le dénouement escamoté est cependant signifiant: sans doute faut-il comprendre qu'Amabed s'est tellement acclimaté aux « agréments » de Rome qu'il met fin à sa correspondance avec son ancien mentor Shastasid ${ }^{110}$.

L'examen d'un court texte, d'abord publié en 1766 dans Le Philosophe ignorant sous le titre de «Petite Digression ${ }^{111}$ », puis significativement ré-intitulé, dans la première édition posthume, publiée à Kehl, des Euvres complètes, "Les Aveugles juges des couleurs $^{112}$ ", peut servir d'épilogue à cette enquête. Dans cette petite fiction, Voltaire détourne la forme du conte pour élaborer une parabole philosophique : alors que les Quinze-Vingts, « dans les commencements », vivaient dans une sorte d'utopie égalitaire et démocratique ( «ils étaient tous égaux »; "leurs petites affaires se décidaient à la pluralité des voix»), l'accession au pouvoir d'un «chef» fait figure d'élément perturbateur qui entraîne toute la communauté dans l'erreur: "Il se mit à juger souverainement des couleurs, et tout fut perdu. »Les conséquences se développent selon un scénario redoublé : un décret (« Il décida que tous les habits des Quinze-Vingts étaient blancs; les aveugles le crurent; ils ne parlaient que de leurs beaux habits blancs, quoiqu'il n'y en eût pas un seul de cette couleur ») suscite la risée de ceux qui voient ("Tout le monde se moqua d'eux »), de même que lorsqu'un " arrêt » est rendu "par lequel tous leurs habits étaient rouges ", alors qu'« il n'y avait pas un habit rouge aux Quinze-Vingts ", " on se moqua d'eux plus que jamais ». De là une "querelle ", enfin apaisée : «[...] on se battit longtemps, et la concorde ne fut rétablie que lorsqu'il fut permis à tous les Quinze-Vingts de suspendre leur jugement sur la couleur de leurs habits. » Mais un ultime rebondissement vient complexifier la portée de la leçon de «cette petite histoire»:

Un sourd, en lisant cette petite histoire, avoua que les aveugles avaient eu tort de juger des couleurs; mais il resta ferme dans l'opinion qu'il n'appartient qu'aux sourds de juger de la musique. 
32 personnages qui incarnent des faiblesses de l'humanité éminemment symboliques: l'aveuglement de ceux qui ne peuvent percevoir la (les) lumière (Lumières); la surdité de ceux qui n'entendent pas le discours, pourtant raisonnable, qui leur est tenu. Cette "petite histoire», qui propose une illustration narrative d'un proverbe attesté en langue (« On dit [...] proverbialement d'un homme qui se mêle de juger des choses dont il n'a aucune connaissance, qu'il en juge comme un aveugle des couleurs $\left.{ }^{113} »\right)$, engage une réflexion sur les limites d'une connaissance qui, selon les principes sensualistes rappelés plus haut, procède des sens : pour un aveugle, spéculer sur l'in-visible revient à se prononcer sur l'in-connaissable. On retrouve par là le discours tenu dans Le Philosophe ignorant au sein duquel le texte, lors de sa publication originale, a le statut de «petite digression » mais qui n'est pas si éloignée du propos théorique défendu: il s'agit à nouveau de pointer, entre autres, la vanité de la métaphysique.

Mais la caractérisation du personnage du "chef » invite à effectuer une interprétation plus spécifique : au-delà de cette fureur absurde de raisonner sur ce qui échappe à la saisie de l'entendement, la parabole dénonce aussi la tyrannie de l'« opinion » dans une histoire qui relate une usurpation d'autorité de la part d'un charlatan devenu «dictateur »: il accède au pouvoir par l'intrigue («il intrigua »), la mise en place d'institutions instaurant une hiérarchie (il «forma d'abord un petit conseil») et la maîtrise de l'argent («il se rendit maître de toutes les aumônes»), et, lorsque sa mainmise sur les esprits («il se fit écouter, [...] il forma des enthousiastes ») se heurte à la contestation des membres de la communauté, il se maintient au pouvoir par la violence (il «les reçut fort mal », "entra en fureur »). Quelques indices suggèrent cependant que la parabole délivre moins un sens politique que religieux : outre que le terme aumônes comporte une acception religieuse attestée, laquelle est encore plus évidente pour le mot enthousiastes ${ }^{114}$, les injures que le « dictateur », qui est issu du rang des "professeurs», profère à l'encontre des récalcitrants («il les traita de novateurs, d'esprits forts, de rebelles, qui se laissaient séduire par les opinions erronées de ceux qui avaient des yeux, et qui osaient douter de l'infaillibilité de leur maître ») ne laissent aucun doute. En somme, le positionnement dogmatique de celui qui, jugeant " souverainement », prétend détenir la vérité au nom de laquelle il s'arroge le droit de combattre les « opinions erronées ", relève de l'intolérance et, dès lors qu'elle conduit au schisme, à la formation de « deux partis » qui s'affrontent au cours d'une incessante "querelle », le fanatisme n'est pas loin. Le texte, écrit dans le contexte de la crispation religieuse consécutive à l'affaire du chevalier de La Barre ${ }^{115}$, se ressent des préoccupations personnelles et militantes du philosophe de Ferney. " philosophiques » majeurs des Romans et contes de Voltaire qui retirent notamment des bénéfices critiques de l'estrangement. Mais, comme l'observent Carlo Ginzburg et avant lui Viktor Chklovski, ce mode opératoire a partie liée avec le genre des devinettes : «Pour voir les choses, il nous faut avant tout les regarder comme si elles étaient parfaitement dénuées de sens - comme des devinettes ${ }^{116}$. " On rejoint par là les analyses précédentes sur la dissémination des indices qui impliquent les lecteurs et lectrices dans le processus herméneutique. L'examen de l'économie des couleurs dans ces textes fait alors apparaître une parenté structurelle entre ce type de dispositif et celui qui repose sur la création et la résolution d'énigmes paramétrées par le «paradigme indiciaire ». 
on content d'instrumentaliser les notations de couleurs dans le cadre d'un projet "philosophique", par l'exploitation d'une rhétorique et d'une symbolique des couleurs, le conteur en effectue des usages narratifs appropriés à l'ancrage générique spécifique de ses récits, tout en mettant en place les conditions d'un questionnement de nature idéologique sur leur valeur culturelle et anthropologique. Voltaire donne ainsi non seulement à voir les couleurs mais aussi à entendre le sens - parfois trompeur dont elles sont porteuses.

\section{NOTES}

1. Dix-huitième siècle, $\mathrm{n}^{\circ} 51$ (La couleur des Lumières), 2019, p. 13-345. Voir, en particulier, l'introduction des éditrices, p. 15-29.

2. B. Maitte, «Les couleurs en physique au $18^{\mathrm{e}}$ siècle : débats autour du renversement de leur statut par Newton ", ibid., p. 93-109.

3. Éléments de la philosophie de Newton, $2^{\mathrm{e}}$ part., chap. 10, en particulier.

4. Micromégas, chap. 2, Romans et contes, 2 vol., F. Deloffre, J. Van den Heuvel et J. Hellegouarc'h (éds), Paris, Gallimard, coll. « Folio classique », 1992, t. I, p. 48.

5. Traité de métaphysique, W. H. Barber (éd.), Les CEuvres complètes de Voltaire (désormais OCV), Oxford, Voltaire Foundation, 1989, t. 14, p. 445-446.

6. Encyclopédie, t. VIII (1765), p.561a. Ici comme ailleurs, l'orthographe des citations a été modernisée.

7. Nouveau Dictionnaire, pour servir de Supplément aux dictionnaires des sciences, des arts et des métiers, Paris, Panckoucke, Stoupe, Brunet, 1776-1777, t. III (1777), p. 567a.

8. La catégorie générique de même que l'étendue des textes que l'on désigne ainsi sont, on le sait, sujettes à débats. Pour une synthèse sur cette question, voir N. Cronk, "The Voltairean Genre of the Conte Philosophique: Does It Exist?», dans P. Robinson (dir.), Enlightenment and Narrative: Essays in Honour of Richard A. Francis by Colleagues and Friends, Nottingham French Studies, $48, n^{\circ} 3$, Automne 2009, p. 61-73. Quelle que soit la part d'arbitraire qui a présidé à la réunion des textes, on s'en tiendra à ceux retenus dans les deux volumes de Romans et contes de Voltaire, ouvr. cité, qui ne comportent pas les contes en vers.

9. Élodie Ripoll analyse les bouleversements esthétiques et symboliques engendrés par l'importance grandissante des notations de couleur dans la fiction romanesque dans la seconde moitié du XVIII ${ }^{\mathrm{e}}$ siècle : voir Penser la couleur en littérature. Explorations romanesques des Lumières au réalisme, Paris, Classiques Garnier, 2018. Voir aussi «La couleur dans le roman des Lumières. Enjeux, emplois et évolutions ", Dix-huitième siècle, $\mathrm{n}^{\circ} 51$ (La couleur des Lumières), 2019, p. 77-92.

10. Certains textes sont même absolument dépourvus de notations de couleurs : la Lettre d'un Turc (1750), l'Histoire d'un bon bramin (1761), Pot-pourri (1765), Aventure indienne (1766).

11. La Princesse de Babylone, chap. 1, 3 et 4, Romans et contes, ouvr. cité, t. I, p. 306-308, 310, 316, 321 et 325. Dans Le Taureau blanc (1773), le cheval d'Amasis, roi de Tanis, est « caparaçonné d'une housse écarlate brochée d'or» (chap. 7, ibid., t. II, p. 262) ; plus loin, « les trois figures d'Isis, d'Osiris et d'Horus, unies ensemble » sont « portées sur un brancard d'or et de pierreries par cent sénateurs de Memphis » (chap. 10, ibid., p. 269). Dans Les Lettres d'Amabed (1769), marque de l'opulence romaine, la princesse de Piombino fait "atteler quatre chars dorés " afin de faire visiter la ville à Amabed et Adaté (Douzième Lettre d'Amabed, ibid., p. 221). 
12. La Princesse de Babylone, chap. 11, ibid., t. I, p. 365 et 368-369.

13. Candide (1759), chap. 2, ibid., t. II, p. 12.

14. Le Monde comme il va, chap. 5, ibid., t. I, p. 70.

15. Ibid.

16. Histoire des voyages de Scarmentado (1756), ibid., p. 173.

17. L'Ingénu (1767), chap. 8, ibid., p. 257-258.

18. Histoire des voyages de Scarmentado (1756), ibid., p. 173.

19. Zadig (1748), « Les disputes et les audiences », ibid., p. 104.

20. Le Monde comme il va, chap. 11, ibid., p. 79.

21. Zadig (1748), « Les combats », ibid., p. 141-144.

22. V. Propp, Morphologie du conte, trad. du russe par C. Ligny, Paris, Gallimard, 1970.

23. Zadig (1748), « La jalousie », Romans et contes, ouvr. cité, t. I, p. 108-109.

24. "Les disputes et les audiences ", ibid., p. 105.

25. " La jalousie », ibid., p. 109.

26. Ibid., p. 109-110.

27. «L'esclavage », ibid., p. 115.

28. «Le basilic ", ibid., p. 136.

29. "Les rendez-vous ", ibid., p. 126. On se souvient que les babouches d'Astarté et de Zadig sont bleues, et que le bonnet de Zadig, tout comme les rubans de la reine, sont jaunes (voir, ci-dessus, n. 23).

30. Carlo Ginzburg (« Signes, traces, pistes. Racines d'un paradigme de l'indice », trad. de l'italien par J.-P. Cottereau, Le Débat, n 6, 1980, p. 3-44, ici p. 23-24) voit dans cet épisode « l'embryon du roman policier $»$.

31. Zadig (1748), " Le chien et le cheval ", Romans et contes, ouvr. cité, t. I.

32. Voir C. Mervaud, « Bestiaires de Voltaire », SVEC 2006:06, Oxford, Voltaire Foundation, 2006, p. $1-200$.

33. Candide (1759), chap. 17, Romans et contes, ouvr. cité, t. II, p. 53.

34. Chap. 18, ibid., p. 61.

35. Nouvelle inadvertance de Voltaire, puisque ce sont cent deux moutons qui sortent d'Eldorado, d'après les chiffres fournis à la fin du chapitre 18.

36. Candide (1759), chap. 20, Romans et contes, ouvr. cité, t. II, p.67. La «friponnerie» de Vanderdendur, le maître du nègre de Surinam, est rapportée au chapitre précédent, p. 64-65.

37. Ibid., p. 68-69.

38. Chap. 24, ibid., p. 83.

39. Chap. 25, ibid., p. 92.

40. Chap. 26, ibid., p. 93.

41. L'Ingénu, chap. 3-4, ibid., t. I, p. 244-245.

42. Candide (1759), chap. 4, ibid., t. II, p. 17.

43. Chap. 8, ibid., p. 26.

44. Chap. 1, ibid., p. 10.

45. Voir U. Eco (dir.), Histoire de la beauté, trad. de l'italien par M. Bouzaher, Paris, Flammarion, 2004.

46. Dans la même veine, on peut songer plus particulièrement au Précis du Cantique des cantiques, « en vers avec le texte» (1759), célébrant la beauté de la Sulamite : les vers de Voltaire évoquent «Les lis, les boutons de rose, / De [s]es deux globes naissants» (v. 4-5); le texte traduit du Cantique des cantiques, un «nez [...] comme la tour du mont Liban qui regarde vers Damas » (OCV, t. 49A, 2010, p. 235 et 244, n. i).

47. Zadig (1748), «Les rendez-vous ", Romans et contes, ouvr. cité, t. I, p. 124-125. On appréciera notamment la dissonance introduite par la comparaison avec « les agneaux sortant du lavoir ». 
48. "Le pêcheur ", ibid., p. 131. Peu avant, accentuant par le burlesque la comparaison entre la couleur de la peau et le lait, elle était dite " plus blanche que ses fromages à la crème » (ibid.).

49. Histoire de Jenni, chap. 4, ibid., t. II, p. 327.

50. Outre le contraste chromatique du blanc et du vermeil, Voltaire reprend un procédé largement éprouvé : l'évocation d'une partie du corps appelle le recours à un adjectif de couleur (ici, le blanc) qui appelle à la suite une comparaison (avec le lait, le lis, l'albâtre, l'hermine). En particulier, la blancheur lactée, d'origine latine, bénéficie d'une longue postérité, au Moyen Âge et au-delà : voir M.-P. Halary, La Question de la beauté et le discours romanesque au début du XIII siècle, Paris, Honoré Champion, 2018, $1^{\text {re }}$ part., chap. 2 (« La codification de la beauté dans les arts poétiques »).

51. La Princesse de Babylone, chap. 1, Romans et contes, ouvr. cité, t. I, p. 311.

52. Chap. 11, ibid., p. 366. On notera en revanche que, "en Huronie ", la beauté de $M^{\text {lle }}$ Abacaba, la maîtresse de l'Ingénu, se mesure au fait que « l'hermine n'est pas plus blanche » (L'Ingénu, ibid., p. 236).

53. Dictionnaire philosophique, C. Mervaud (éd.), OCV, t. 35, 1994, p. 409-410.

54. La Princesse de Babylone, chap. 10, Romans et contes, ouvr. cité, t. I, p. 362.

55. Micromégas, chap. 3, ibid., p. 49.

56. Jeannot et Colin (1764), ibid., p. 223.

57. Candide (1759), chap. 1, ibid., t. II, p. 11. On retrouve plus tard, sous le nom de Paquette, ce personnage qui se prostitue à Venise.

58. Zadig (1748), « Les yeux bleus », ibid., t. I, p. 159-160.

59. Candide (1759), chap. 11, ibid., t. II, p. 33.

60. Chap. 29, ibid., p. 103.

61. L'une et l'autre paraissent accomplir la prophétie de Memnon qui croit que la sagesse consiste à ne jamais aimer de femme car, affirme-t-il, « en voyant une beauté parfaite, je me dirai à moi-même : "Ces joues-là se rideront un jour ; ces beaux yeux seront bordés de rouge ; cette gorge ronde deviendra plate et pendante ; cette belle tête deviendra chauve." " (Memnon [1748], ibid., t. I, p. 162)

62. La blancheur de Mélinade est soulignée, lors de son apparition dans le récit, par son brillant équipage constitué de « six grand chevaux blancs » (Le Crocheteur borgne, ibid., p. 28).

63. Ibid., p. 29-30.

64. Ibid., p. 30.

65. Ibid., p. 31.

66. Le Taureau blanc, chap. 1, ibid., t. II, p. 237. Le narrateur précise que « celui de Pasiphaé, celui dont Jupiter prit la figure pour enlever Europe, n'approchait pas de ce superbe animal » (ibid.).

67. Chap. 11, ibid., p. 272.

68. C'est encore l'une des caractéristiques du roi des Scythes dont sont évoqués les «bras nus, aussi nerveux que blancs» (La Princesse de Babylone, chap. 1, ibid., p. 305). Dans Le Blanc et le Noir (1764), le narrateur précise aussi que Topaze est «beau, bien fait, blanc comme une Circassienne » (ibid., p. 203).

69. Voir, ci-dessus, n. 65.

70. Histoire de Jenni, chap. 4, Romans et contes, ouvr. cité, t. II, p. 327.

71. La Princesse de Babylone, chap. 1, ibid., t. I, p. 306. On verra plus loin qu'il en va de même des dignitaires ecclésiastiques romains.

72. L'Ingénu, chap. 1, ibid., p. 233.

73. Candide (1759), chap. 8, ibid., t. II, p. 27-28.

74. Chap. 14, ibid., p. 45. La couleur vermeil est aussi un marqueur de l'opulence, par exemple dans L'Homme aux quarante écus (1768), lorsque le personnage, amaigri, sort de son cachot : « [...] je rencontrai un homme joufflu et vermeil dans un carrosse à six chevaux [...]. Son maître d'hôtel, 
aussi vermeil que lui, avait deux mille francs d'appointements [...]. » (« Désastre de l'homme aux quarante écus ", ibid., p. 112)

75. Histoire de Jenni, chap. 1, ibid., t. II, p. 311.

76. On doit renoncer l'examen des nombreuses mentions du rougissement des personnages, femmes et hommes, qui conduirait à des conclusions similaires. Sur cette question, voir J.C. Abramovici, " "Commander le silence à sa physionomie": la rougeur des hommes ", Dixhuitième siècle, $\mathrm{n}^{\circ} 51$ (La couleur des Lumières), 2019, p. 304-319.

77. Zadig (1748), « L'ermite », Romans et contes, ouvr. cité, t. I, p. 145 et 149.

78. Le Blanc et le Noir, ibid., p. 203.

79. Ibid., p. 203-204.

80. Ibid., p. 212.

81. Ibid., p. 213.

82. Ibid., p. 213-214.

83. Ibid., p. 214-215. On se souvient que Le Blanc et le Noir paraît dans le recueil des Contes de Guillaume Vadé, et que Voltaire s'emploie dans divers textes à accréditer l'existence fictive d'une famille Vadé dont les membres lui servent occasionnellement de pseudonymes : voir O. Ferret, "Vade mecum, vade retro: le recours au pseudonyme dans la démarche pamphlétaire voltairienne ", La Lettre clandestine, $\mathrm{n}^{\circ}$ 8, 2000, p. 65-82.

84. Voir l'épisode de "L'envieux» dans Zadig: Zadig compose des vers impromptus, brise en deux sa tablette et jette les deux morceaux dans un buisson; l'envieux met la main sur une moitié et non seulement "chaque moitié de vers qui remplissait la ligne faisait un sens ", mais «par un hasard encore plus étrange, ces petits vers se trouvaient former un sens qui contenait les injures les plus horribles contre le roi ». Alors que Zadig est perdu, le perroquet du roi retrouve l'autre moitié, collée à une pêche, qu'il porte sur les genoux du monarque : après réassemblage, « on lut alors les vers tels que Zadig les avait faits» (ibid., p. 97-98).

85. Pour une autre approche de cette question, voir G. Linte, «La couleur de la peau dans le discours médical (1730-1770)», Dix-huitième siècle, $\mathrm{n}^{\circ} 51$ (La couleur des Lumières), 2019, p. 261-274.

86. La Princesse de Babylone, chap. 11, Romans et contes, ouvr. cité, t. I, respectivement p. 371, 374 et 372 .

87. L'Homme aux quarante écus, ibid., t. II, p. 136.

88. Candide (1759), chap. 11, ibid., p. 35.

89. Chap. 14, ibid., p. 43. Martin insiste sur cette origine lorsqu'il évoque "un valet métis » (chap. 24, p. 83).

90. Histoire de Jenni, chap. 9, ibid., p. 356.

91. Histoire des voyages de Scarmentado, ibid., t. I, p. 178. Scarmentado commente : « On n'avait rien à répliquer à un discours si sage. " (ibid.)

92. Expression employée dans le chapitre 152 («Des îles françaises, et des flibustiers ») de l'Essai sur les mœurs, qui évoque avec indignation le traitement réservé aux "esclaves nègres ou mulâtres » : « Nous leur disons qu'ils sont hommes comme nous, qu'ils sont rachetés du sang d'un Dieu mort pour eux, et ensuite on les fait travailler comme des bêtes de somme, on les nourrit plus mal ; s'ils veulent s'enfuir, on leur coupe une jambe, et on leur fait tourner à bras l'arbre des moulins à sucre lorsqu'on leur a donné une jambe de bois ; après cela nous osons parler du droit des gens. » (OCV, t. 26A, 2013, p. 285)

93. Candide (1759), chap. 19, Romans et contes, ouvr. cité, t. II, p. 62-63.

94. Les Lettres d'Amabed, Septième Lettre et Quatrième Lettre d'Amabed, ibid., respectivement p. 213 et $209-210$.

95. Voir le chapitre que Michèle Duchet consacre à Voltaire dans Anthropologie et histoire au siècle des Lumières [1971], Paris, Albin Michel, 1995; J.-M. Moureaux, "Race et altérité dans l'anthropologie voltairienne», dans S. Moussa (dir.), L'idée de "race» dans les sciences et la 
littérature (XVIII et XIX siècles), Paris, L'Harmattan, coll. « Histoire des sciences humaines », 2003, p. 41-53.

96. Voir, ci-dessus, n. 19 et 20.

97. Voir, ci-dessus, n. 16 ; voir aussi Candide (1759), chap. 3, Romans et contes, ouvr. cité, t. II, p. 16.

98. Chap. 5, ibid., p. 22.

99. Histoire de Jenni, chap. 2, ibid., p. 314.

100. Les Lettres d'Amabed, Seconde Lettre d'Adaté, ibid., p. 189-190.

101. La Princesse de Babylone, chap. 10, ibid., t. I, p. 360-361. Les jésuites sont des « ex-druides » car ce conte, publié en 1768 , est postérieur à l'expulsion de leur ordre.

102. Ibid., chap. 9, respectivement p. 356-357 et 355. Les éditeurs traduisent : «Par saint Martin, quel beau garçon ! Par saint Pancrace, quel bel enfant ! »

103. Ibid., p. 357-358.

104. C. Ginzburg, À distance. Neuf essais sur le point de vue en histoire, chap. 1, «L'estrangement. Préhistoire d'un procédé littéraire ", trad. de l'italien par P.-A. Fabre, Paris, Gallimard, 2001, p. 15-36. Carlo Ginzburg reprend dans une perspective historicisée une notion que le formaliste Viktor Chklovski, dans son entreprise de définition de «l'art» - en général - «comme procédé », désigne en russe par ostranienie (ibid., p. 15-16). Le traducteur s'explique sur le choix de traduire par ce terme, emprunté à Antoine Vitez, l'italien straniamento (ibid., n. *, p. 15).

105. Les Lettres d'Amabed, Sixième Lettre d'Adaté, Romans et contes, ouvr. cité, t. II, p. 201. Les souliers portés aux mains désignent les gants dont la locutrice, venue des Indes, ignore l'usage. Amabed rapporte de son côté que, lors de son arrivée dans la «ville de Roume " en compagnie d'Adaté et de sa suivante Déra, « un homme vêtu d'une robe violette, accompagné de deux autres en manteau noir, vint [les] féliciter sur [leur] arrivée » (Onzième Lettre d'Amabed, ibid., p. 219). Le personnage est ensuite désigné comme « l'homme violet » (Douzième Lettre d'Amabed, ibid., p. 220).

106. Seizième Lettre d'Amabed, ibid., p. 227.

107. Vingtième Lettre d'Amabed, ibid., p. 233.

108. Dix-septième Lettre d'Amabed, ibid., p. 230. Il était question plus haut de la rencontre avec ce personnage de «théologien du feu vice-Dieu » qui sert d'introducteur aux usages de la cour de Rome, présenté, en raison des réponses pour le moins hétérodoxes qu'il apporte aux questions d'Amabed, comme «bien dessalé »: «Je me forme beaucoup avec lui, et je me sens déjà tout autre ", écrit Amabed (Quatorzième Lettre d'Amabed, ibid., p. 223-224).

109. Vingtième Lettre d'Amabed, ibid., p. 234.

110. Une évolution discrète du personnage se dessine au fil de ses lettres qui trace en pointillés les étapes de sa formation entamée au moment de la Quatorzième Lettre (voir, ci-dessus, n. 108) et rappelée dans l'avant-dernière: "Nous prenons en patience tous les agréments qu'on nous fait goûter dans ce pays-ci. Chaque jour nous apprend des choses dont nous ne nous doutions pas. On se forme beaucoup par les voyages. " (Dix-neuvième Lettre, ibid., p. 232-233) Le retour n'est dès lors plus envisageable.

111. Petite Digression, ibid., t. I, p. 226-227.

112. Cuvres complètes de Voltaire, [Kehl], De l'Imprimerie de la Société littéraire-typographique, 1784,70 vol., t. 45, p. $449-450$.

113. Dictionnaire de l'Académie française, éd. 1762, art. « Aveugle ».

114. Ibid., art. "Enthousiaste »: "Visionnaire, fanatique. [...] On appelait ainsi certains hérétiques qui se croyaient inspirés. »

115. On se souvient que le jeune homme de 19 ans, accusé de blasphème, est exécuté à Abbeville le $1^{\mathrm{er}}$ juillet 1766 : un exemplaire du Dictionnaire philosophique, trouvé à son domicile lors d'une perquisition, est brûlé sur le bûcher. Voir M. Méricam-Bourdet (dir.), "Autour de l'affaire La Barre ", Revue Voltaire, n 17 (L'affaire La Barre), 2017, p.9-178, ainsi que les "Inédits et documents », p. 179-259. 


\title{
RÉSUMÉS
}

Quoique la catégorie générique des « contes philosophiques » soit sujette à débats, le traitement dans les Romans et contes de Voltaire du motif de la couleur invite à penser l'articulation dans ces textes du narratif et du philosophique. Centrée sur les personnages de récits mettant en jeu une rhétorique et une symbolique des couleurs, l'étude montre comment Voltaire exploite leurs possibilités narratives pour instaurer avec les lecteurs et lectrices un jeu herméneutique. Elle s'intéresse aussi à la manière dont le traitement des représentations topiques de la beauté problématise la valeur symbolique des couleurs ainsi que leur ancrage genré. Elle examine enfin comment la mise en œuvre de la différence des couleurs ouvre la voie à une réflexion philosophique au sens large, qui met en crise le manichéisme, inclut des considérations anthropologiques sur la diversité humaine, mais aussi, par la satire antichrétienne, permet l'expression d'une philosophie militante qui rejoint les combats poursuivis dans d'autres types de textes.

Although the generic category of contes philosophiques is a matter of discussion, the literary treatment of the motif of color in Voltaire's Romans et contes invites us to think how the narrative and philosophical dimensions of these texts should be articulated. Focusing on the characters of a number of stories that bring into play a rhetoric and a symbolism of colors, the study shows how Voltaire exploits their narrative potential to set up a hermeneutic game with the reader. It also looks at how the treatment of stereotyped representations of beauty can question the symbolic value of colors including from a gendered point of view. Finally, it examines how the implementation of the difference of colors opens the way to a philosophical reflection in the broadest sense, which puts Manichaeism into crisis, involves anthropological concerns about human diversity, but also, through anti-Christian satire, enables the expression of a committed philosophical discourse that matches the struggles carried out in other types of texts.

\section{INDEX}

Mots-clés : Voltaire, contes, couleurs, symbolique, herméneutique, caractérisation genrée, manichéisme, valeur anthropologique, colonialisme, satire antichrétienne

Keywords : Voltaire, tales, color, symbolism, hermeneutics, gendered characterization, Manichaeism, anthropological value, colonialism, anti-Christian satire

\author{
AUTEUR \\ OLIVIER FERRET \\ Université Lumière Lyon 2, IHRIM UMR 5317 \\ Olivier.Ferret@univ-lyon2.fr
}

